\title{
Cytomegalovirus-Associated Intussusception with Florid Vascular Proliferation in an Infant
}

\author{
Heejung Park · Sanghui Park ${ }^{1}$. Young Ju Hong ${ }^{2} \cdot$ Sun Wha Lee ${ }^{3} \cdot$ Min-Sun Cho $^{1}$ \\ Department of Pathology, National Health Insurance Service Ilsan Hospital, Goyang; Departments of ${ }^{1}$ Pathology, ${ }^{2}$ Surgery, and ${ }^{3}$ Radiology, \\ Ewha Womans University School of Medicine, Seoul, Korea
}

Intussusception is usually idiopathic, with no pathologic lead point except for the presence of reactive lymphoid hyperplasia, which is probably associated with gastrointestinal infection or a reaction to newly introduced food proteins. Viral infections, such as those caused by non-enteric adenovirus, human herpes virus (HHV)-6, HHV-7, and Epstein-Barr virus, are known etiologic factors of intussusception. Cytomegalovirus (CMV)-associated intussusception has been reported rarely; there have been three case reports in immunocompetent and human immunodeficiency virus-infected infants. ${ }^{1-3}$ None of these three case reports described a detailed histologic pattern, except ischemic necrosis of the small intestine due to a prolonged clinical history and delayed surgery. Here, we report a case of a CMV-associated inflammatory polyp with unique gross and microscopic findings as a leading cause of ileoileal intussusception in an 8-week-old healthy female.

\section{CASE REPORT}

An 8-week-old healthy female infant was admitted to the hospital following 10 episodes of repeated vomiting and poor oral intake for one day. She was born by normal vaginal delivery at 40 weeks without any perinatal problems and with a birth weight of $3.98 \mathrm{~kg}$. At admission, her height was $63.1 \mathrm{~cm}$ and her body weight was $6 \mathrm{~kg}$, which was $89.4 \%$ of her ideal body

Corresponding Author

Min-Sun Cho, M.D.

Department of Pathology, Ewha Womans University Mokdong Hospital, 1071

Anyangcheon-ro, Yangcheon-gu, Seoul 158-710, Korea

Tel: +82-2-2650-5044, Fax: +82-2-2650-2789, E-mail: mcho1124@ewha.ac.kr

Received: February 5, 2015 Revised: March 25, 2015

Accepted: April 1, 2015 weight. Her body temperature was $37.2^{\circ} \mathrm{C}$. The results of the laboratory examination were unremarkable. Ultrasonography (US) on admission showed ileocolic intussusception at the hepatic flexure of the colon, which was successfully reduced by US-guided saline reduction. However, vomiting continued, although at a reduced rate, and bloody diarrhea developed intermittently. Follow-up US the next day revealed a newly developed ileoileal intussusception, which persisted until additional follow-up US (Fig. 1A). Five days after symptoms developed, an exploratory laparotomy and segmental resection of the ileum was performed.

The resected ileum contained a roughly ovoid, sessile, polypoid mass, measuring $4 \times 2 \times 1 \mathrm{~cm}$. On the mucosal side of the mass, an aggregation of multiple small round polyps was observed, which had a cobblestone-like or nodule-aggregating appearance (Fig. 1B). The cut surface revealed multiple round-toovoid solid nodules with focal hemorrhages scattered from the submucosal to the subserosal connective tissue (Fig. 1C).

Upon histological examination, each mucosal polyp appeared to represent variably expanded and fused mucosal folds of the plicae circulares (Fig. 1D). The surface villi were flattened, multifocally, and covered with fibrinopurulent exudate. The crypts were irregularly deformed and cystically dilated. The lamina propria showed exuberant granulation tissue-type small vessel proliferation and fibroblastic reaction, with inflammatory infiltrates composed of plasma cells, small and large lymphoid cells, eosinophils, and histiocytes (Fig. 2A). The muscularis propria were partly disorganized and fused with the muscularis mucosae, suggesting a stretched intestinal wall of intussusception. The deeper layer showed multiple well-defined solid nodules, in which proliferated florid vessels were intermixed with fibroblasts and inflammatory cell infiltrates (Fig. 2B). Some of the nodules 

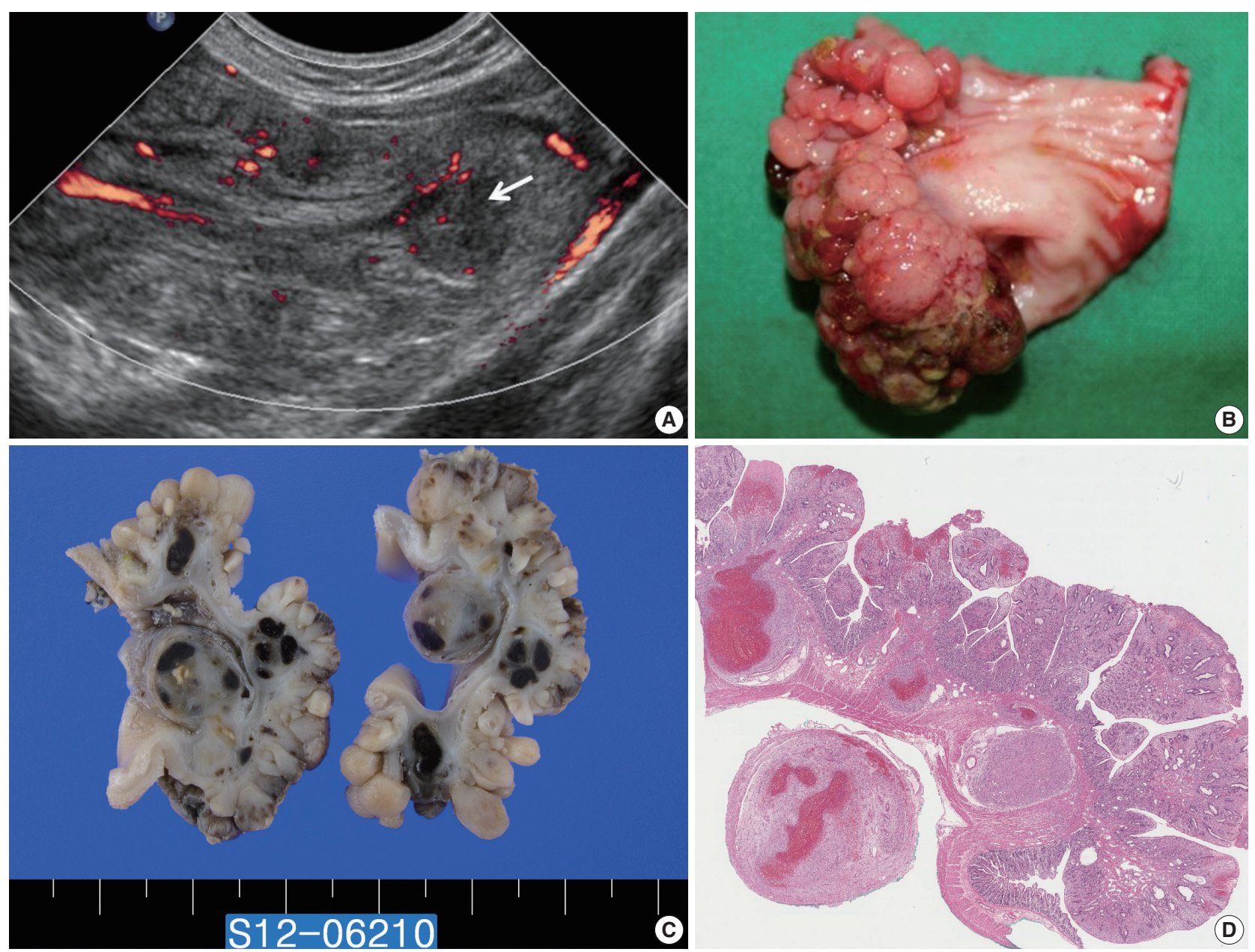

Fig. 1. (A) Longitudinal ultrasonography of the lower abdomen with a Doppler study shows an ovoid mass with alternating thick hypoechoic and thin hyperechoic layers, indicating ileoileal intussusception and Doppler flow signals at the intussusceptum. A round hypoechoic lesion (arrow) indicating a lead point of intussusception is identified. (B) The ileum reveals a $4 \times 2 \times 1 \mathrm{~cm}$, roughly ovoid, sessile, polypoid mass with a conglomerated nodular or nodule-aggregating appearance. (C) The cut surface shows thickened mucosa and multiple round solid nodules with focal hemorrhages at deeper layers. (D) The polyp is composed of enlarged plicae circulares having dilated and distorted crypt glands with expanded lamina propria.

showed multifocal hemorrhages with fibroblastic proliferation, resembling reorganizing thrombus (Fig. 2C). Careful searching revealed that a few capillary endothelial cells and stromal cells exhibited characteristic eosinophilic intranuclear and cytoplasmic viral inclusions beneath and around the eroded mucosal surface in the focal area (Fig. 2D). These cells displayed positive immunostaining for CMV. After surgery, the CMV antigen was not detected in peripheral blood but was isolated from a stool culture in additional laboratory tests.

\section{DISCUSSION}

CMV infection occurs throughout the gastrointestinal tract, with ulceration as the most common morphologic change.,
Gastrointestinal CMV infection presenting as a polyp is unusual, and only a few cases have been reported. ${ }^{2,6,7}$ The lesions exhibit features like those of inflammatory or juvenile polyps, including surface ulceration, distention and shape irregularity of the crypt glands, and granulation tissue-type small capillary proliferation with inflammatory cell infiltration. Therefore, based on the dominant histologic pattern in the biopsy, $\mathrm{CMV}$-associated polyps could be variably diagnosed as inflammatory fibroid polyps, inflamed hyperplastic polyps, inflammatory myofibroblastic tumors or even vascular tumors, etc. ${ }^{2,6,7}$ In our case, the polyp had histologic features reminiscent of a juvenile polyp. However, glandular change and the preserved plicae circulares structure did not fit the diagnosis of a juvenile polyp. Thorough microscopic observation identified cells with CMV inclusion bodies. 

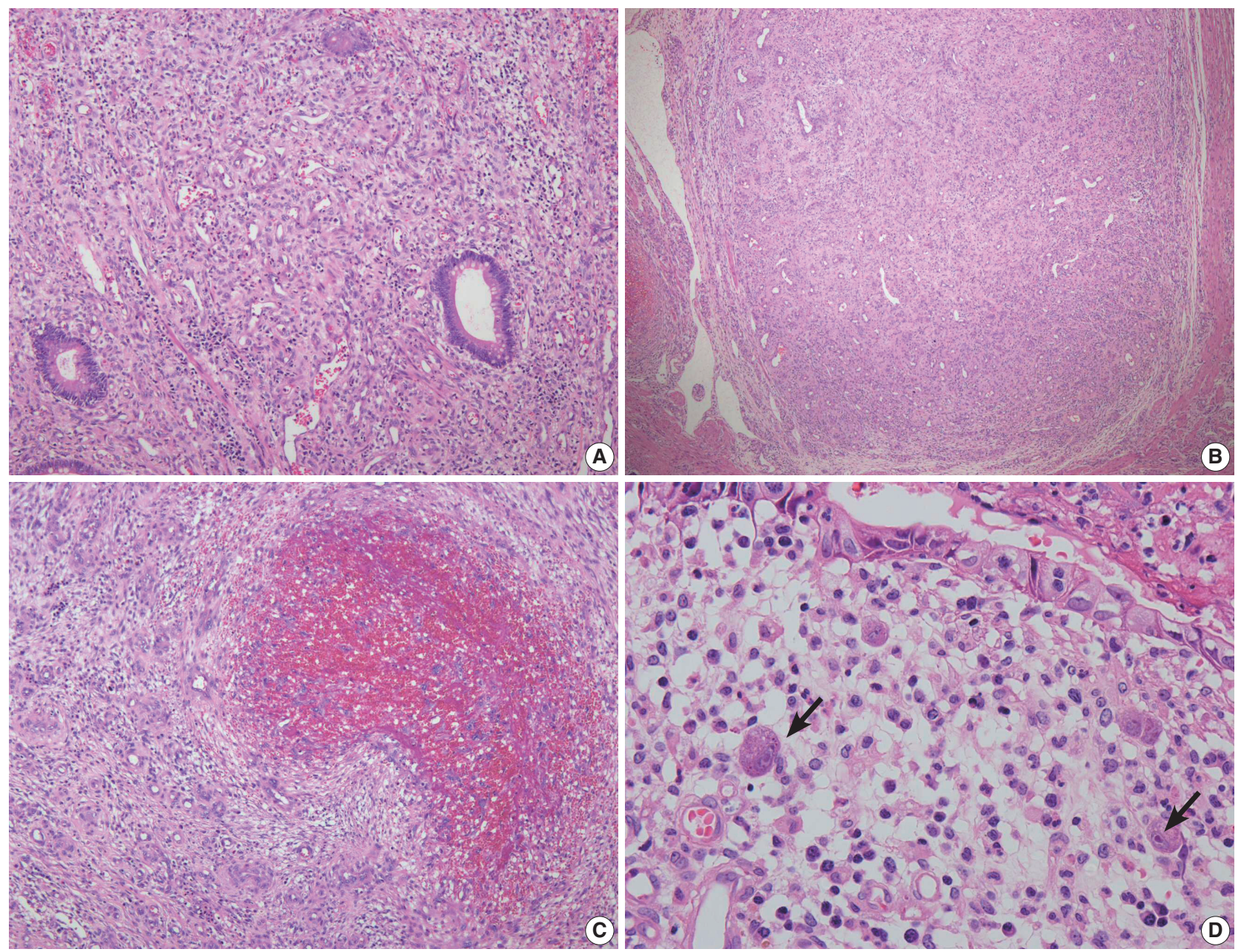

Fig. 2. (A) The lamina propria shows granulation tissue-type small vessel proliferation. (B) The nodules at deeper layers are composed of proliferated florid small vessels and fibroblasts. (C) Organizing-thrombus-like areas are noted focally in the nodules. (D) A few stromal cells with intranuclear and cytoplasmic cytomegalovirus inclusions are present beneath the eroded mucosal surface.

Recurrent intussusception produces variable nonspecific histologic changes, including disorganization of the muscularis propria, fusion of the muscularis mucosae with the muscularis propria, focal submucosal fibrosis, telangiectasia, fibrous serosal adhesion, localized mucosal hyperplasia, etc. ${ }^{8}$ In addition, florid small vascular proliferation has been reported, which may be so pronounced as to raise the possibility of primary vascular neoplasm. ${ }^{9,10}$ Although the mechanisms underlying the development of such vascular lesions are difficult to ascertain, repeated mechanical forces applied to the bowel wall during long-term mucosal prolapse associated with intussusception trigger angiogenesis, resulting in an exuberant form of highly vascularized granulation tissue. The clinical history of prolonged intussusceptions may explain the granuloma pyogenicum-like solid nodules scattered at deeper layers with focal hemorrhages, and the organizing thrombus-like features of this case.
In summary, it is considered that CMV-induced mucosal inflammation acted as a lead point of intussusception, and persistent prolonged intussusception caused nodular florid vascular proliferation of deeper layers, in this case with unique microscopic and gross findings.

\section{Conflicts of Interest}

No potential conflict of interest relevant to this article was reported.

\section{Acknowledgments}

This study was supported by a grant from the Memorial Research Foundation for the 50th anniversary of the Medical College of Ewha Womans University, Medical College of Ewha Womans University Alumnae Association. 


\section{REFERENCES}

1. Ma R, Alam M, Pacilli M, Hook E, Nagy A, Carroll D. A rare case of neonatal intussusception caused by cytomegalovirus. Eur J Pediatr Surg 2014; 24: 184-6.

2. Agaimy A, Mudter J, Markl B, Chetty R. Cytomegalovirus infection presenting as isolated inflammatory polyps of the gastrointestinal tract. Pathology 2011; 43: 440-6.

3. Zupancic JA, Pennie RA, Issenman R. Intussusception in a child with cytomegalovirus infection. Pediatr Infect Dis J 1994; 13: 548-9.

4. Chetty R, Roskell DE. Cytomegalovirus infection in the gastrointestinal tract. J Clin Pathol 1994; 47: 968-72.

5. Seo TH, Kim JH, Ko SY, et al. Cytomegalovirus colitis in immunocompetent patients: a clinical and endoscopic study. Hepatogastroenterology 2012; 59: 2137-41.

6. Kelesidis T, Tozzi S, Mitty R, Worthington M, Fleisher J. Cytomegalovirus pseudotumor of the duodenum in a patient with AIDS: an unrecognized and potentially treatable clinical entity. Int J Infect Dis 2010; 14: e274-82.

7. Lin WR, Su MY, Hsu CM, et al. Clinical and endoscopic features for alimentary tract cytomegalovirus disease: report of 20 cases with gastrointestinal cytomegalovirus disease. Chang Gung Med J 2005; 28: 476-84.

8. Noffsinger A, Fenoglio-Preiser CM, Maru D, Gilinsky N. Gastrointestinal diseases: atlas of nontumor pathology. Washington, DC: American Registry of Pathology, 2008.

9. Ramsden KL, Newman J, Moran A. Florid vascular proliferation in repeated intussusception mimicking primary angiomatous lesion. J Clin Pathol 1993; 46: 91-2.

10. Bavikatty NR, Goldblum JR, Abdul-Karim FW, Nielsen SL, Greenson JK. Florid vascular proliferation of the colon related to intussusception and mucosal prolapse: potential diagnostic confusion with angiosarcoma. Mod Pathol 2001; 14: 1114-8. 